\title{
Enzyme Replacement Therapy Clears Gb3 Deposits from a Podocyte Cell Culture Model of Fabry Disease but Fails to Restore Altered Cellular Signaling
}

\author{
Fabian Braun ${ }^{a, b, c, d} \quad$ Linda Blomberg ${ }^{a, b, c} \quad$ Susanne Brodesser ${ }^{b} \quad$ Max C. Liebau ${ }^{a, b, c, e}$ \\ Bernhard Schermer ${ }^{a, b, c, f} \quad$ Thomas Benzing ${ }^{a, b, c, f} \quad$ Christine E. Kurschat ${ }^{a, b, c}$

\begin{abstract}
aDepartment II of Internal Medicine and Center for Rare Diseases Cologne, University Hospital of Cologne, Cologne, Germany, ${ }^{b}$ Cologne Excellence Cluster on Cellular Stress Responses in AgeingAssociated Diseases (CECAD), University of Cologne, Cologne, Germany, 'Center for Molecular Medical Center Hamburg-Eppendorf, Hamburg, Germany, ${ }^{e}$ Department of Pediatrics and Center for Molecular Medicine Cologne, University of Cologne, Faculty of Medicine and University Hospital Cologne, Cologne, Germany, 'Systems Biology of Aging, University of Cologne, Cologne, Germany
\end{abstract} \\ Medicine Cologne, University of Cologne, Cologne, Germany, dIII. Department of Medicine, University
}

\author{
Key Words \\ Fabry $\bullet$ Podocyte $・$ Enzyme Replacement Therapy
}

\begin{abstract}
Background/Aims: Fabry disease (FD) is a lysosomal storage disorder characterized by impaired alpha-galactosidase $A(\alpha-G a l$ ) enzyme activity due to mutations in the GLA gene. While virtually all tissues are affected, renal damage is particularly critical for the patients' outcome. Currently, powerful diagnostic tools and in vivo research models to study FD in the kidney are lacking, which is a major obstacle for further improvements in diagnosis and therapy. The present study focuses on the effects of enzyme replacement therapy on a previously established podocyte cell culture model of Fabry disease. Methods: We investigated the effect of in vitro application of $\alpha$-Gal A on Fabry podocytes for 3 days, mimicking enzyme replacement therapy. We studied reduction of $\mathrm{Gb3}$ levels and dysregulated molecular pathways such as autophagy, mTOR/AKT signaling and pro-fibrotic signaling by employing immunofluorescence, electron microscopy, tandem mass spectrometry and western blot. Results: We detected complete resolution of $\mathrm{Gb} 3$ accumulation in Fabry podocytes upon $\alpha-G a l$ A treatment. Despite robust Gb3 clearance, dysregulation of the signaling pathways investigated was not reversed. Conclusion: This study presents first evidence for Gb3independent effects regarding dysregulation of signal transduction mechanisms in FD not recovering upon $\alpha$-Gal A treatment. We assume that intracellular alterations observed in FD

F. Braun and L. Blomberg contributed equally to this work.

Prof. Dr. Christine Kurschat,

$\mathrm{MD}$

Department II of Internal Medicine, University Hospital Cologne

Kerpener Str. 62, 50937 Cologne (Germany)

Tel. +49 221478 4480, Fax +49 221479 5959, E-Mail christine.kurschat@uk-koeln.de
\end{abstract}




\section{Cellular Physiology Cell Physiol Biochem 2019;52:1139-1150 \\ \begin{tabular}{ll|l} 
aOl: 10.33594/000000077 & $\begin{array}{l}\text { O 2019 The Author(s). Published by } \\
\text { Cell Physiol Biochem Press GmbH\&Co. KG }\end{array}$
\end{tabular} \\ Braun et al.: Enzyme Replacement Therapy in a Podocyte Cell Culture Model of Fabry \\ Disease}

may have a point of no return after which a reversal of dysregulated cellular signal transduction by $\alpha$-Gal A treatment is not effective, despite Gb3 clearance. Our observations suggest further research on signal transduction mechanisms altered in Fabry podocytes and on determining the appropriate time for initiation of Fabry therapy.

(c) 2019 The Author(s). Published by Cell Physiol Biochem Press GmbH\&Co. KG

\section{Introduction}

Fabry disease (FD) [OMIM: 301500] is an X-linked hereditary disorder caused by mutations in the alpha-galactosidase A $(G L A)$ gene resulting in an impairment of glycosphingolipid metabolism. Due to loss or defective function of the enzyme alphagalactosidase $\mathrm{A}(\alpha-\mathrm{Gal} \mathrm{A})$ the terminal $\alpha$-glycosidic bond of galactose cannot be cleaved from the sphingolipid globotriaosylceramide (Gb3), thus resulting in accumulation of Gb3 within the lysosome. This defect and the subsequent lysosomal dysfunction can be detected in virtually every cell in every tissue [1]. The clinical presentation encompasses a broad spectrum of symptoms ranging from gastrointestinal dysfunction, hypohidrosis, heat sensitivity and fever to severe neuropathic pain, ischemic stroke at very young age, cardiomyopathy and renal insufficiency [2]. While hemizygous male patients typically display severe symptoms, the clinical manifestations seen in heterozygous female patients can comprise the whole range of clinical features from the absence of symptoms to very severe pathologies as seen in male patients with classical FD. Premature death mostly occurs due to cardiac complications and end-stage renal disease (ESRD) [2].

Renal insufficiency is caused in part by progressive Gb3 depositions primarily affecting podocytes, terminally differentiated cells with limited potential of recovery and regeneration $[3,4]$. First renal symptoms, consequently, are (micro-) albuminuria and combined proteinuria at later stages, rarely coinciding with the development of hypertension. Subsequently, the histological phenotype is characterized by progressive glomerulosclerosis and nephron loss leading to tubular atrophy and interstitial fibrosis [2]. Due to the multitude of unspecific symptoms the majority of Fabry patients are diagnosed late in the course of their disease after organ damage has already occurred.

To date, treatment of Fabry disease relies on either intravenous enzyme replacement therapy or, in the case of amenable mutations, oral chaperone administration $[5,6]$. Both approaches have been shown to ameliorate clinical symptoms and disease progression, with limited potential to reverse already established organ damage.

Since the current murine models for Fabry disease differ significantly from the glomerular phenotype seen in human disease [7-10], our group established an immortalized human podocyte cell culture model with robust $\alpha$-Gal A activity reduction and subsequent Gb3 accumulation [11]. Molecular characterization demonstrated dysregulated autophagy as a probable result of decreased mTOR and AKT phosphorylation and protein abundance.

In this study, we describe the successful use of a compound for enzyme replacement therapy, agalsidase alfa, to clear Gb3 deposits in our established podocyte cell culture model for Fabry disease. Interestingly, despite Gb3 clearance, we did not detect any effect of enzyme replacement therapy on dysregulated autophagy, increased mTOR activation or other, already published molecular alterations such as increased Notch1 activation or dysregulated TGF beta signaling in Fabry podocytes. These findings suggest yet unknown disease mechanisms independent of $\mathrm{Gb3}$ accumulation and warrant further research for additional therapeutic targets of renal dysfunction in Fabry disease.

\section{Materials and Methods}

Human podocyte cell culture

Conditional immortalized human podocytes and podocytes with reduced $\alpha$-Gal activity were cultured as previously described [11]. Briefly, immortalized podocytes were stably transduced with co-shRNA 


\section{Cellular Physiology Cell Physiol Biochem 2019;52:1139-1150 \\ \begin{tabular}{ll|l} 
and Bin 10.33594/000000077 & C 2019 The Author(s). Published by
\end{tabular} \\ Published online: 17 April $2019 \quad$ Cell Physiol Biochem Press GmbH\&Co. KG \\ Braun et al.: Enzyme Replacement Therapy in a Podocyte Cell Culture Model of Fabry \\ Disease}

(control) and shRNA 894 (reducing $\alpha$-galactosidase A activity to 2-4\%, Fig. S1 - for all supplemental material see www.cellphysiolbiochem.com), cultured in RPMI media (Sigma-Aldrich, Taufkirchen, Germany) with insulin-transferrin-sodium selenite as supplement (ThermoFisher Scientific, Waltham, USA) containing $10 \%$ fetal bovine serum (Biochrom, Berlin, Germany). Cells proliferated at $33^{\circ} \mathrm{C}$ until they reached a confluence of $60-70 \%$. Subsequently, cells were differentiated at $37^{\circ} \mathrm{C}$ for 14 days before harvesting them for further analyses. Cells were regularly tested for mycoplasm infection using the mycoplasm detection kit from Minerva biolabs (Minerva Biolabs, Berlin, Germany).

\section{Treatment with $\alpha$-Gal A}

Agalsidase alfa (Shire, Berlin, Germany) was used at a final concentration of $250 \mathrm{nM}$. A portion of the provided agalsidase alfa was heat-inactivated at $95^{\circ} \mathrm{C}$ for 30 minutes to serve as a negative control. Chemical chaperone deoxygalactonojirimycin hydrochlorid (DGJ, Sigma-Aldrich, Taufkirchen, Germany) was added at a final concentration of $10 \mu \mathrm{M}$ to stabilize the enzyme under cell culture conditions. Cells were treated with agalsidase alfa at Day 8, 10 and 12 of differentiation.

\section{Stress Induction via Interleukin $1 \beta$}

For western blot analysis of mTOR and AKT phosphorylation, cells were treated with interleukin $1 \beta$ at a concentration of $10 \mathrm{ng} / \mathrm{ml}$ for one hour prior to western lysate preparation.

\section{Immunofluorescence}

After 7 days of differentiation podocytes were seeded onto coverslips to a confluency of $80 \%$. Cells were treated with $\alpha$-Gal A according to the above mentioned protocol for the following 7 days at $37^{\circ} \mathrm{C}$. After fixation with 4\% PFA and blocking with normal donkey serum in PBS containing 0.1\% Triton-X100, coverslips were incubated with rat anti-Gb3 (CD77) antibody (Abcam, ab19795) o/n at $4^{\circ} \mathrm{C}$. Secondary antibody staining was performed for 1 hour at room temperature with Cy3-conjugated goat anti-rat IgM $\mu$ chain specific (Jackson, 112-165-020). Coverslips were mounted in ProLong Diamond with DAPI (ThermoFischer, P36962) and imaged with a ZEISS LSM710 confocal microscope (Zen software).

\section{RT-qPCR}

Cells were lysed and total RNA was isolated (TRI Reagent, Sigma Aldrich). RNA concentration was measured (Nanodrop 1000 spectrophotometer, Peqlab) and cDNA was synthesized from $1000 \mathrm{ng}$ RNA template using the High Capacity cDNA RT Kit (Applied Biosystems) according to the supplier's protocol. Dilutions were made for RT-qPCR analysis to determine mRNA expression levels which were normalized against a reference gene. The Power SYBR Green PCR Master Mix (Applied Biosystems) was used to multiply and measure the cDNA with a 7900HT Fast Real-time PCR System (Applied Biosystems). The following PCR program was used: $95^{\circ} \mathrm{C}$ for $10 \mathrm{~min}$, followed by 40 cycles of $15 \mathrm{~s}$ at $95^{\circ} \mathrm{C}, 60 \mathrm{~s}$ at $60^{\circ} \mathrm{C}$, then $15 \mathrm{~s}$ at $95^{\circ} \mathrm{C}$ followed by a melt of the product from $60^{\circ} \mathrm{C}-95^{\circ} \mathrm{C}$. The primer sequences (IDT) used are: hACTB forward 5'-GGACTTCGAGCAAGAGATGG, hACTB reverse 5'-AGCACTGTGTTGGCGTACAG, hHPRT1 forward 5'-TGACACTGGCAAAACAATGCA, hHPRT1 reverse 5'-GGTCCTTTTCACCAGCAAGCT, hGLA forward 5'-TGGAAGGATGCAGGTTATGAG, hGLA reverse 5'-CCCTAGCTTCAGTCCTTTGCT, hHES1 forward 5'-ACCAAAGACAGCATCTGAGCA, hHES1 reverse 5'-GCCGCGAGCTATCTTTCTTCA, hCOL4 forward 5'-CTCCTGGTTCTCCACAGTCAG, hCOL4 reverse 5'-AAGACCCTGCCAGACCAAGG, hFN1 forward 5'-GGTCCGGGACTCAATCCAAAT, hFN1 reverse 5'-ACCCTGAACTGTAAGGGTTCTT, hTGF $\beta 1$ forward 5'-CCCAGCATCTGCAAAGCTC, hTGF 31 reverse 5'-GTCAATGTACAGCTGCCGCA, hHPRT1 forward 5'-TGACACTGGCAAAACAATGCA, hHPRT reverse 5'-GGTCCTTTTCACCAGCAAGCT. The $\triangle \triangle C$ C method was used for statistical analysis to determine relative gene expression levels.

\section{Enzyme assay}

Cultured human podocytes were collected in cold PBS. Cell lysis was performed by freezing and thawing over five cycles. Enzyme activity for $\alpha$-Galactosidase A ( $\alpha$-Gal) and $\beta$-Galactosidase $(\beta$-Gal) was measured after incubation of lysed cells with 4-methylumbelliferyl a-D-galactopyranoside for $\alpha$-Gal or 4-methylumbelliferyl b-D-galactopyranoside for $\beta$-Gal (Sigma, Taufkirchen, Germany) as described previously [12]. $\alpha$-Gal A activity was normalized to $\beta$-Gal activity, functioning as an internal control. 


\section{Cellular Physiology Cell Physiol Biochem 2019;52:1139-1150

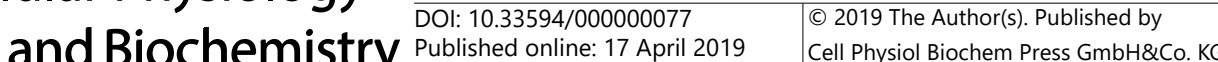 \\ Braun et al.: Enzyme Replacement Therapy in a Podocyte Cell Culture Model of Fabry \\ Disease}

Western blot

Size separation was done using SDS-PAGE. Samples were blotted onto polyvinylidene difluoride membranes and visualized with enhanced chemiluminescence after incubation of the blots with corresponding antibodies anti-phospho AKT (S473), anti-AKT, anti-phospho-mTOR (S2448), anti-mTOR, anti-phospho-p38 (Thr180/Tyr182), anti-p38, anti- phospho-p42/44 (Thr 202/Tyr204), anti-p42/44) (Cell Signaling, Danvers, MA, USA); anti- $\beta$-catenin (BD, Heidelberg, Germany); anti- $\beta$-tubulin (Developmental Studies Hybridoma Bank, University of Iowa, USA), Notch1 cleaved Val (Abcam, Cambridge, UK). For quantification of band intensity, individual bands of at least three independent experiments were quantified using ImageStudio lite software (Li-Cor, Lincoln, USA). The intensity of every band is expressed as ratio to the corresponding loading control.

\section{Gb3 quantification}

Gb3 levels in podocytes were determined by Liquid Chromatography coupled to Electrospray Ionization Tandem Mass Spectrometry (LC-ESI-MS/MS): Cells were harvested in ice cold PBS and centrifuged at $3.000 \mathrm{~g}, 4^{\circ} \mathrm{C}$ for $5 \mathrm{~min}$. Supernatants were removed and cell pellets frozen at $-20^{\circ} \mathrm{C}$. After thawing cells were homogenized in water $\left(10^{7}\right.$ cells / $\left.100 \mu \mathrm{l}\right)$ using the Precellys 24 Homogenisator (Peqlab, Erlangen, Germany). The protein content of the homogenate was routinely determined using bicinchoninic acid. To $100 \mu \mathrm{l}$ of homogenate $750 \mu \mathrm{l}$ of methanol/chloroform 2:1 (v/v) and $200 \mathrm{pmol}$ of the deuterated internal standard D3-Gb3 C18:0 (Matreya, State College, PA, USA) were added. Lipids were extracted overnight at 48 ${ }^{\circ} \mathrm{C}$. Interfering glycerolipids were degraded by alkaline hydrolysis adding $75 \mu \mathrm{l}$ of $1 \mathrm{M}$ potassium hydroxide in methanol and incubating for $2 \mathrm{~h}$ at $37^{\circ} \mathrm{C}$. After neutralization with glacial acetic acid, the lipid extract was desalted by reversed phase C18 (RP-18) chromatography. Dried lipid extracts were resolved in $200 \mu \mathrm{l}$ of the LC mobile phase solvent (see below) and sonicated for $5 \mathrm{~min}$. After centrifugation $\left(12,000 \times \mathrm{g}, 5 \mathrm{~min}, 4^{\circ} \mathrm{C}\right.$ ), $40 \mu \mathrm{l}$ of the clear supernatants were transferred to auto-injector vials. LC-MS/MS analysis was performed using a Core Shell Kinetex C8 column, $100 \times 2.1 \mathrm{~mm}$ ID, $2.6 \mu \mathrm{m}$ particle size, $100 \AA ̊$ pore size (Phenomenex, Aschaffenburg, Germany) with detection using a QTRAP 6500 triple quadrupole/linear ion trap mass spectrometer (SCIEX, Darmstadt, Germany). The LC (1260 Infinity Binary LC System; Agilent, Waldbronn, Germany) was operated isocratically at a flow rate of $500 \mu \mathrm{l} / \mathrm{min}$ with a mobile phase of $0.1 \%$ formic acid and $2 \mathrm{mM}$ ammonium acetate in methanol. $20 \mu \mathrm{l}$ of sample were injected. The total run time was $3 \mathrm{~min} . \mathrm{Gb3}$ species were monitored in the positive ion mode using their specific Multiple Reaction Monitoring (MRM) transitions of the $[\mathrm{M}+\mathrm{H}]^{+}$precursor ion to the product ion $\mathrm{m} / \mathrm{z} 264.4$ (quantifiers) and to the product ion generated by the loss of one molecule of water and the three hexose residues (qualifiers). The instrument settings for nebulizer gas (Gas 1), turbogas (Gas 2), curtain gas, and collision gas were 60 psi, 65 psi, 35 psi, and medium, respectively. The Turbo V ESI source temperature was $350{ }^{\circ} \mathrm{C}$, and the ionspray voltage was $5500 \mathrm{~V}$. For all MRM transitions the values for declustering potential, entrance potential, and cell exit potential were $210 \mathrm{~V}, 10 \mathrm{~V}$, and $8 \mathrm{~V}$, respectively. The collision energies ranged from 36 to $68 \mathrm{~V}$. The Gb3 quantifier peaks were integrated using the Analyst 1.6.2 software (AB SCIEX). Endogenous Gb3 species were quantified on the basis of external calibration curves which were calculated from LC-MS/MS measurements of serially diluted synthetic Gb3 standard solutions prepared in the mobile phase solvent within the range of 0.0 to $190 \mathrm{pmol}$ on column. To each dilution a fixed amount of the internal standard D3-Gb3 C18:0 was added. The standard calibration curves were plotted based on molar concentration versus peak area ratio of Gb3 standards to D3-Gb3 C18:0. Linearity and correlation coefficients (R2) of the calibration curves were obtained via linear regression analysis. R2 of the calibration curves were $>0.99$. The calculated amounts of endogenous Gb species were normalized to the protein content of the sample.

\section{Electron microscopy}

Cells were grown on collagen-coated melamine foil before start of $\alpha$-Gal A treatment. After 7 days of treatment, cells were washed in PBS and fixed in 2\% Paraformaldehyde, 2\% Glutaraldehyde in 0, 1M sodium cacodylate buffer for 30 minutes at room temperature and 30 minutes at $4^{\circ} \mathrm{C}$. After washing and incubation in $1 \%$ osmium tetroxide cells were dehydrated in rising ethanol and embedded in epoxy resin. Ultra thin slices of 70nm were done at the Ultracut (Leica Systems, Wetzlar, Germany). Contrast enhancement was achieved through incubation in 1,5\% uranylacetate and leadnitrate. Analysis and images were done at the JEM-2100 PlusTransmission Electron Microscope (JEOL, Tokio, Japan). 


\section{Cellular Physiology Cell Physiol Biochem 2019;52:1139-1150 \\ \begin{tabular}{ll|l} 
and Biochemisty $10.33594 / 000000077$ & (c) 2019 The Author(s). Published by \\
Cell Physiol Biochem Press GmbH\&Co. KG
\end{tabular} \\ Braun et al.: Enzyme Replacement Therapy in a Podocyte Cell Culture Model of Fabry \\ Disease}

\section{Results}

$\alpha$-Gal A treatment for three days clears Gb3 deposits in human podocytes with reduced expression of alpha-galactosidase

We used the human podocyte cell culture model for Fabry disease published previously [11]. This cell culture model is characterized by reduced levels of GLA mRNA and functional enzyme, as well as Gb3 accumulation (Fig. S1a-c). As previously described [11], this phenotype results in dysregulated autophagy detected by increased LC3-II expression and lower mTOR and AKT phosphorylation in podocytes with reduced $\alpha$-Gal A activity (Fig. S1d-e). We started treatment of control and $\alpha-G a l$ A deficient podocytes after seven days of differentiation. Active or inactivated $\alpha$-Gal A, both including 1-deoxygalactonojirimycin (DGJ), was administered every other day for a total amount of 3 treatments. DGJ is an iminosugar also known as migalastat. We used this compound to stabilize $\alpha$-Gal A enzyme activity in vitro. Migalastat has recently been licensed for the treatment of Fabry disease in patients with GLA mutations leading to $\alpha$-Gal A folding defects amenable to migalastat treatment. Immunofluorescence staining (IF) for Gb3 deposition depicted a marked reduction in fluorescent signal in Fabry podocytes treated with active $\alpha$-Gal A (Fig. 1A). This effect was abolished entirely upon heat inactivation of the enzyme (Fig. 1A). To confirm the results in IF and to quantify Gb3 reduction by $\alpha$-Gal A treatment we quantified Gb3 levels by Liquid Chromatography coupled to Tandem Mass Spectrometry (LC-MS/MS). Likewise, we detected a significant decrease of total Gb3 content down to control levels after $\alpha$-Gal A treatment, while Gb3 accumulation prevailed when cells were treated with inactivated enzyme (Fig. 1B). These findings were confirmed by LC-MS/MS analysis of Gb3 subspecies differing in the fatty acyl chain lengths (Fig. 1C).

\section{Gb3 clearance upon $\alpha$-Gal A treatment does not ameliorate dysregulated autophagy}

After having established a successful protocol for in vitro enzyme replacement therapy resulting in almost complete clearance of Gb3 in our cell culture model, we analyzed previously described Fabry-associated alterations in autophagy regulation. We expected to reverse dysregulated autophagy by $\alpha$-Gal A treatment. Surprisingly, despite almost complete clearance of Gb3 from $\alpha$-Gal A-deficient podocytes by treatment with $\alpha$-Gal A, we did not observe a decrease in LC3-II expression when compared to untreated Fabry podocytes or podocytes treated with heat-inactivated enzyme (Fig. 2A). To investigate cellular ultrastructure we performed electron microscopy (Fig. 2B). Zebra bodies, a correlate of accumulated storage material, were present in untreated Fabry podocytes. We did not detect any zebra bodies in actively treated $\alpha$-Gal A-deficient podocytes, indicating clearance of Gb3 by treatment. Despite $\alpha$-Gal A administration, overrepresentation of autophagosomes was still present (Fig. 2B). Examining the total abundance and phosphorylation of mTOR and AKT proteins under stress conditions (IL $1 \beta$ treatment), we detected significant effects of reduced Gb3 accumulation only on AKT abundance (Fig. 2C, D), and merely a tendency to augmented mTOR abundance and mTOR/AKT phosphorylation. Interestingly, treatment with DGJ and inactivated enzyme resulted in a significant increase of both AKT/mTOR abundance and phosphorylation even though this condition depicts high levels of Gb3. These results might point to a chaperone-mediated effect on AKT/mTOR. To determine whether the dysregulated autophagy in $\alpha$-Gal A-deficient podocytes is due to transcriptional changes, we investigated the expression of two genes involved in autophagy initiation, Beclin 1 (BCN1) and GABA Type A Receptor-Associated Protein (GABARAP) [13]. As expected we detected increased expression in Fabry podocytes compared to control cells. This effect remained present despite enzyme replacement therapy and subsequent Gb3 depletion (Fig. 2E, F).

$\alpha$-Gal A impairment results in activation of the Noch1 and TGF beta cascades

Sanchez-Niño et al. described activation of Notch1 and TGF beta pathways in cultured human podocytes by increased concentrations of circulating lyso-Gb3 [14, 15]. We were 


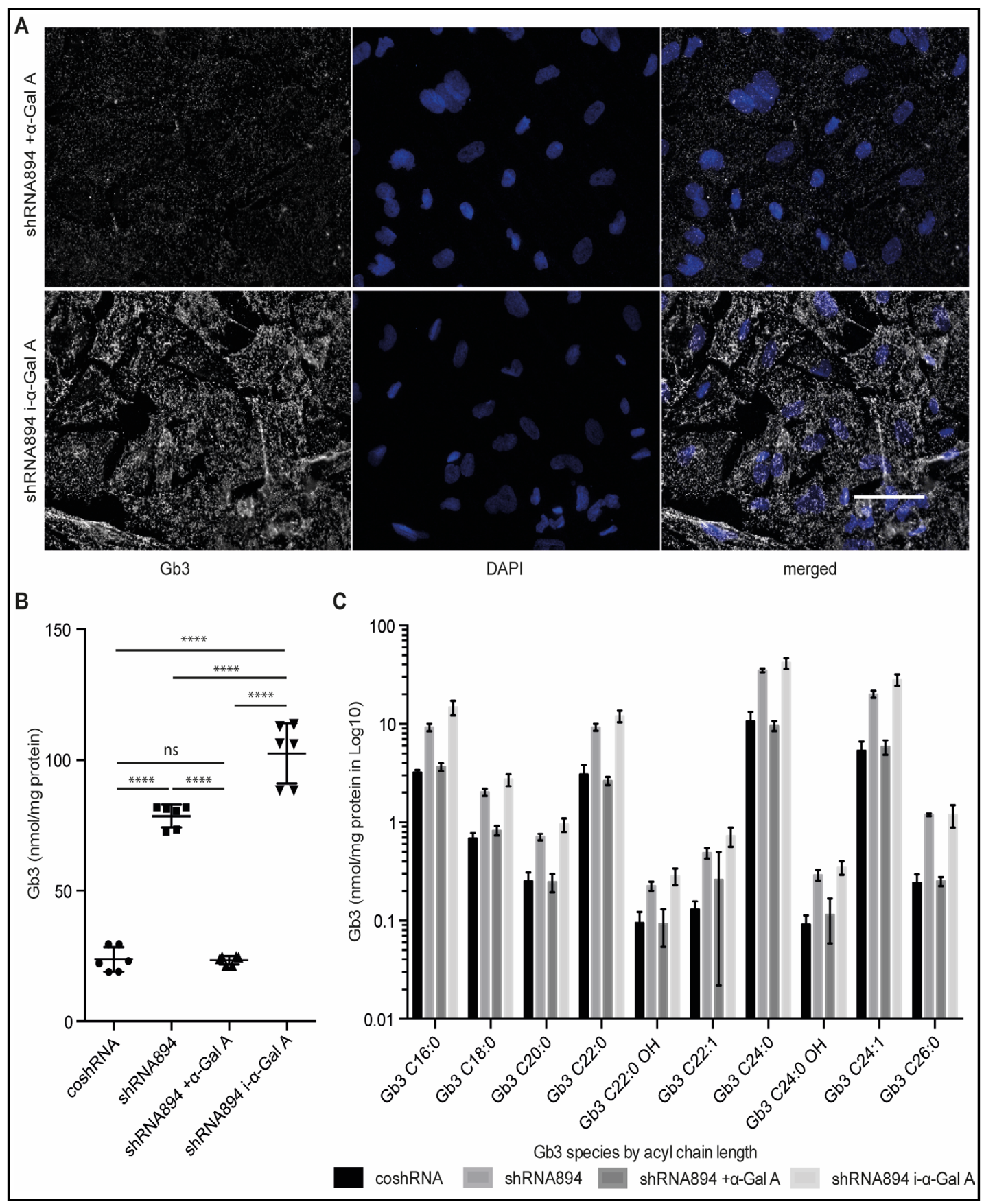

Fig. 1. Gb3 detection in $\alpha$-Gal A-deficient podocytes at baseline and under ERT. (A) Immunofluorescence of Gb3 (gray), counterstained with DAPI (blue) of control (coshRNA) and $\alpha$-Gal A-deficient podocytes (shRNA894) (scale bar $50 \mu \mathrm{m}$ ). (B) Quantification of total Gb3 content measured by LC-MS/MS normalized to total protein levels in control (coshRNA), $\alpha$-Gal A-deficient podocytes (shRNA894) and $\alpha$-Gal A-deficient podocytes treated with active $(+\alpha-G$ al $A)$ or inactivated $(i-\alpha-G a l A)$. Bars depict mean $\pm S D, * * * *=p<0,0001$. (C) Quantification of Gb3 subspecies measured by LC-MS/MS normalized to total protein levels in control (coshRNA), $\alpha$-Gal A-deficient podocytes (shRNA894) and $\alpha$-Gal A-deficient podocytes treated with active $(+\alpha-G a l A)$ or inactivated $(i-\alpha-G a l A)$. Bars depict mean $\pm S D$, significance levels not indicated. 

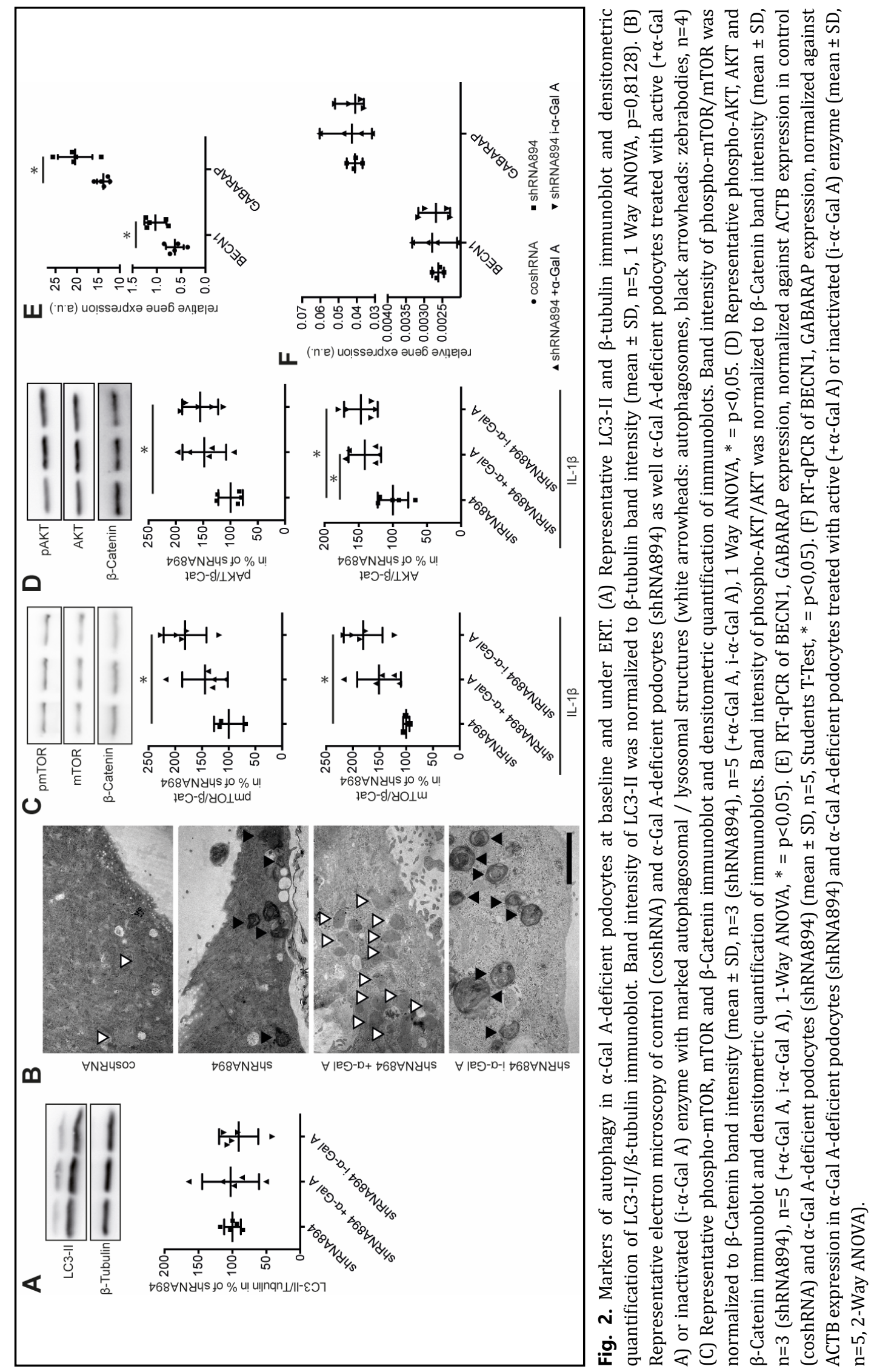


\section{Cellular Physiology Cell Physiol Biochem 2019;52:1139-1150

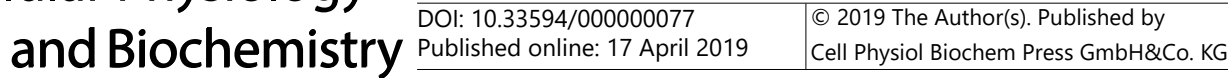

Fig. 3. Profibrotic signaling in $\alpha$-Gal A-deficient podocytes at baseline and under ERT. (A) Representative cleaved Notch1 and $\beta$-tubulin immunoblot and densitometric quantification of cleaved Notch1/ß-tubulin immunoblot. Band intensity of cleaved Notch1 was normalized to $\beta$-tubulin band intensity (mean $\pm S D, n=3$, Students t-test, ${ }^{*}=\mathrm{p}<0,05$ ). (B) RT-qPCR of HES, Col4A4, FN1 expression, normalized against ACTB expression in control (coshRNA) and $\alpha$-Gal A-deficient podocytes (shRNA894) (mean \pm SD, $\mathrm{n}=5$, Students T-Test, $*=$ $\mathrm{p}<0,05$ ). (C) Representative cleaved Notch1 and $\beta$-tubulin immunoblot and densitometric quantification of cleaved Notch1/ß-tubulin immunoblot. Band intensity of cleaved Notch1 was normalized to $\beta$-tubulin band intensity (mean \pm SD, $\mathrm{n}=3$, 1-Way ANOVA). (D) RTqPCR of HES, Col4A4, FN1

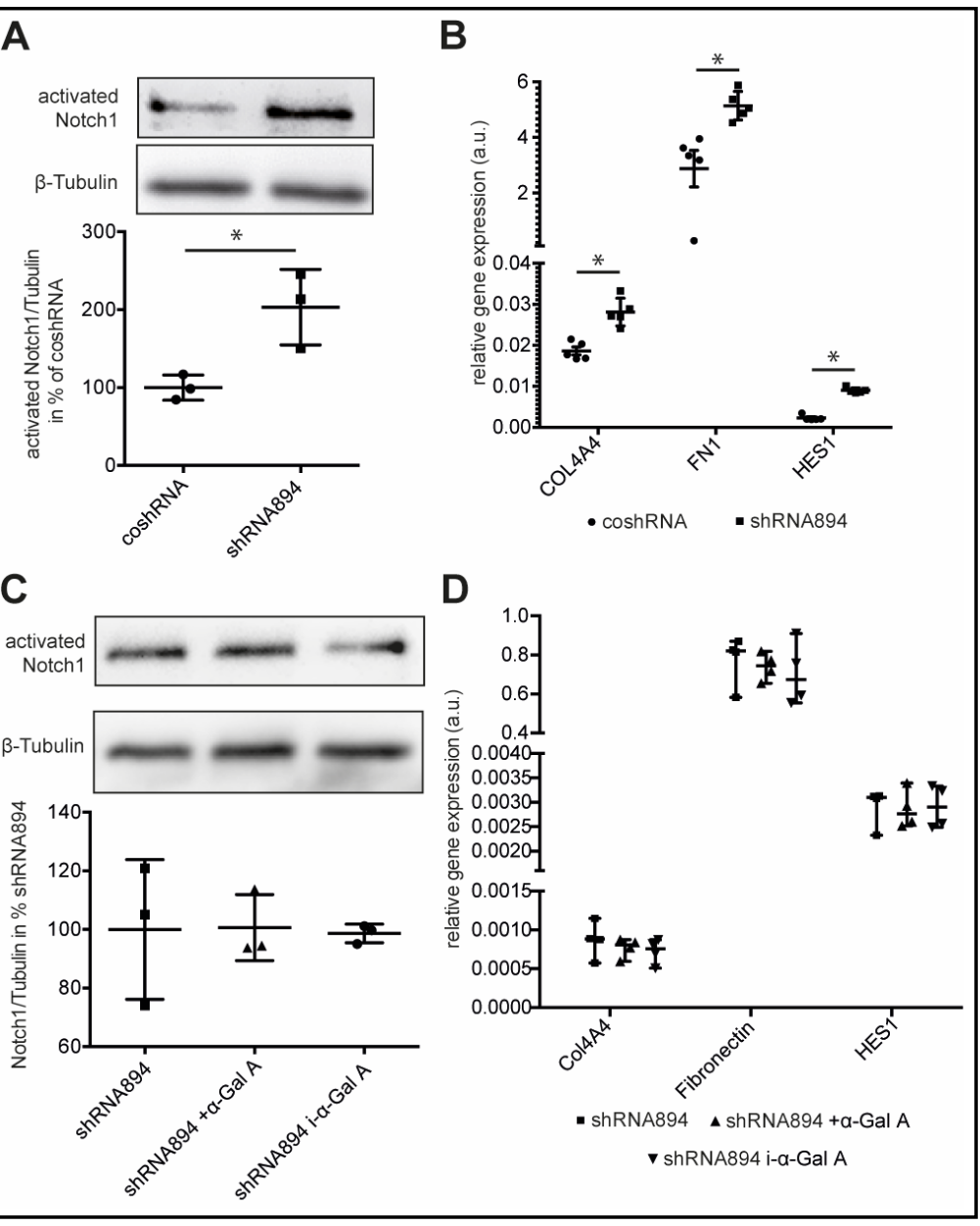
expression, normalized against ACTB expression in $\alpha$-Gal A-deficient podocytes (shRNA894) and $\alpha$-Gal A-deficient podocytes treated with active $(+\alpha-G a l A)$ or inactivated $(i-\alpha-G a l A)$ enzyme (mean $\pm S D, n=4$, 2-Way-ANOVA).

able to confirm their results in our model by detecting enhanced cleavage of Notch1 in $\alpha$-Gal A-deficient podocytes (Fig. 3A) and, subsequently, higher expression of target genes fibronectin (FN1) and Hes Family BHLH Transcription Factor 1 (HES1) as well as augmented transcription of TGF beta target Collagen 4A4 (Col4A4) (Fig. 3B). When investigating the influence of Gb3 depletion upon $\alpha$-Gal A treatment on Notch1, Col4A4, FN1 and HES1, again, we did not find an amelioration of the phenotype in $\alpha$-Gal A-treated podocytes (Fig. 3C, D).

\section{Discussion}

This study presents the first report of successful and rapid Gb3 clearance by enzyme replacement therapy (ERT) in a human podocyte cell culture model of Fabry disease. The employed protocol resulted in complete removal of accumulated Gb3 down to control cell levels (Fig. 1) after only three treatment cycles of $\alpha$-Gal A and DGJ. DGJ was used to stabilize $\alpha$-Gal A activity in vitro. The $\alpha$-Gal A concentration used in our cell culture experiments represents about four times the amount of $\alpha$-Gal A present in a patient's circulation with five liters of blood volume directly after agalsidase alfa infusion at a concentration of $0.2 \mathrm{mg} / \mathrm{kg}$ body weight. We used a higher $\alpha$-Gal A concentration in vitro since most cell culture models exhibit high levels of resilience to pharmacological compounds and, subsequently, higher 


\section{Cellular Physiology Cell Physiol Biochem 2019;52:1139-1150 \\ \begin{tabular}{ll|l} 
and Biochemisty $10.33594 / 000000077$ & (c) 2019 The Author(s). Published by \\
Cell Physiol Biochem Press GmbH\&Co. KG
\end{tabular} \\ Braun et al.: Enzyme Replacement Therapy in a Podocyte Cell Culture Model of Fabry \\ Disease}

concentrations of compounds are usually needed to cause treatment effects in vitro [16]. In our Fabry podocyte model $\alpha$-Gal A treatment demonstrates a high efficacy in clearance of Gb3 deposits in vitro. We observed no Gb3 clearance after $\alpha$-Gal A heat inactivation, even though DGJ was still present in this experimental setup. DGJ, also known as migalastat, is capable of enhancing enzyme activity by stabilization of the enzyme, especially in GLA mutations leading to folding defects of the protein structure. Our cell culture model is characterized by a reduction of GLA gene expression by small hairpin RNAs. Therefore, the remaining cellular enzyme activity (2-4\%, Fig. S1) is due to the residual expression of a conventionally folded and normally functioning protein. The remaining activity is not enhanced by DGJ in our model, consistent with only a mild increase in normal $\alpha$-Gal A activity upon DGJ administration [17]. A clear effect of migalastat on Gb3 accumulation in podocytes in the context of amenable mutations, analyzed by EM of patient renal biopsies, has previously been reported [18].

Strikingly, Gb3 clearance from our podocyte cell culture model did not result in the normalization of the autophagy machinery (Fig. 2A). Electron microscopy depicted numerous autophagosomes still present in $\alpha$-Gal A-treated Fabry podocytes. The absence of $\alpha$-Gal A treatment effects on dysregulated autophagy in our model might be due to the shorter period of therapy we employed in this study. A decrease of LC3 staining in kidney biopsies after three years of enzyme replacement therapy was reported in 2010, presenting immunofluorescence images of glomeruli [19]. Clear evidence that decreased LC3 immunofluorescence mirrored amelioration of autophagic flux in podocytes was not provided. Our data suggest that mere replacement of active enzyme and subsequent rapid decrease of intracellular Gb3 deposits is not sufficient to directly improve dysregulated autophagy in our podocyte model.

We observed a tendency to increased mTOR and AKT expression as well as mTOR and AKT phosphorylation upon treatment with $\alpha$-Gal A and DGJ (Fig. 2C, D). In the context of heatinactivated $\alpha$-Gal A employment +DGJ this difference was significant. This observation may point towards a chaperone-dependent pleiotropic effect, since DGJ was present while $\alpha$-Gal A was inactive. We hypothesize that decreased binding of DGJ to the denatured protein may lead to this more pronounced effect. Still, DGJ treatment alone did not result in restoration of dysregulated autophagy activity. Increased transcription of critical genes involved in autophagy initiation was present in Fabry podocytes (fig. 2E). BECN1 and GABARAP represent two members of the Coordinated Lysosomal Expression and Regulation (CLEAR) network [20] with Transcription Factor EB (TFEB) being its most prominent transcription factor. TFEB, a master regulator of lysosome integrity and autophagy, is known to play a role in multiple lysosomal storage diseases such as Gaucher disease and Niemann-Pick disease, and is currently investigated as a potential therapeutic target in Pompe disease [21-23]. TFEB function also affects podocyte biology [24]. A recent study by our group investigating primary urine cells of Fabry patients identified additional proteins of the CLEAR network to be more abundant in Fabry patients compared to healthy individuals [25].

Profibrotic signaling has been investigated in different models of Fabry disease in the past. We were able to recapitulate enhanced cleavage of Notch1 in our Fabry podocyte model exhibiting endogenous accumulation of $\mathrm{Gb3}$, previously published in a podocte cell culture system upon administration of exogenous lyso-Gb3 (Fig. 3A, B) [15]. However, treatment with active enzyme and subsequent Gb3 clearance could not abrogate enhanced Notch1 cleavage and expression of TGF-beta targets in our model (Fig. 3C, D). The activation of profibrotic signaling through $\alpha$-Gal A deficiency and Gb3 accumulation could very well underlie a selfsupplying loop in which an initial stimulus triggers a cascade of events that cannot be stopped by simply abolishing the stimulus. Additionally, accumulation of Gb3 might not be the only pathophysiological stimulus leading to enhanced profibrotic signaling in Fabry disease.

The presented study is limited by its in vitro setup and the experimental way $\alpha$-Gal A deficiency is achieved. In our model, $\alpha$-Gal A activity is reduced by inhibition of protein transcription due to small hairpin RNAs. Additional intracellular effects resulting from translation of a defective enzyme and a dysregulated unfolded protein response with ER stress in Fabry patient podocytes cannot be mirrored in our model. Further research should focus on studying reported classical and non-classical GLA gene mutations to investigate additional 


\section{Cellular Physiology Cell Physiol Biochem 2019;52:1139-1150

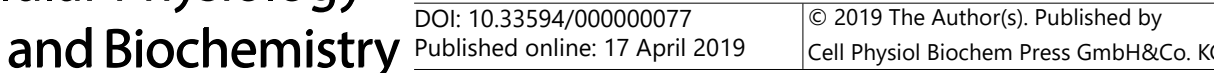 \\ Braun et al.: Enzyme Replacement Therapy in a Podocyte Cell Culture Model of Fabry \\ Disease}

or differing effects of ERT and migalastat therapy. Likewise, the analysis of different cell types, preferably the ones that can be kept in culture longer than immortalized differentiated human podocytes [26], would add valuable information to whether the presented results are cell type-dependent or independent and whether they can be influenced by prolonging overall time of treatment with ERT or migalastat.

Our study provides the first in vitro results that supplementation of $\alpha$-Gal A enzyme activity and subsequent $\mathrm{Gb} 3$ clearance are not sufficient to rescue profibrotic signaling and dysregulated autophagy in a podocyte cell culture model of Fabry disease. Long-term studies on the effect of ERT on organ dysfunction have shown that current therapeutic approaches in Fabry disease patients can slow down disease progression but do not significantly reverse damage that has already occurred $[27,28]$. Short-term results of chaperone therapy also depict some but not complete improvement of organ function after 1.5 years of treatment [29]. Therefore, distinct but thus far incompletely characterized disease mechanisms may not be reversed with currently available therapy. Since our data points to additional, yet unknown disease mechanisms and a point of no return despite Gb3 clearance, these findings may underscore that early treatment initiation is crucial in Fabry disease patients to prevent irreversible cellular damage [30]. New diagnostic strategies employing the advances in mass spectrometry [31-33] as well as initiating kidney biopsies in Fabry disease patients earlier in the course of the disease have already been suggested [34-37].

The use of (lyso)-Gb3 as a biomarker for Fabry disease activity and for monitoring therapy needs to be critically investigated since additional mechanisms besides Gb3 accumulation-induced cellular damage may be present in Fabry disease [18, 31]. Regular assessments of eGFR, proteinuria and cardiac fibrosis have been reported to be superior prognostic markers in a recent study [38].

\section{Conclusion}

In conclusion, we provide the first evidence for enzyme replacement therapy-induced and Gb3 clearance in a podocyte cell culture model in vitro. Surprisingly, Gb3 removal failed to improve dysregulated autophagy and a profibrotic phenotype providing first molecular evidence for Gb3-independent disease mechanisms warranting intensified research and identification of additional molecular pathways leading to Fabry disease pathology.

\section{Acknowledgements}

We acknowledge excellent technical support provided by Beatrix Martiny. The authors have no ethical conflicts to disclose. This work was supported by a Gerok-Position [to F. B.] and an MD scholarship [to L. B.] provided by the Medical Faculty of the University of Cologne. B. S. was supported by the German Research Foundation (SCHE 1562/6). ERT was kindly provided by Shire, Berlin, Germany. The funding organizations as well as Shire had no influence on the design, outcome or discussed conclusions of the presented study.

The research was designed by F. B. and C. E. K. F. B., L. B., and S. B. conducted the experiments and acquired the data. F. B., L. B. and C. E. K. analyzed the data. F. B., L. B., M. C. L., B. S., T. B. and C. E. K. wrote the manuscript. All authors approved the final version of the paper. F. B. and L. B. contributed equally to this paper.

\section{Disclosure Statement}

F. B. received travel support of Amicus Therapeutics. C. K. received travel support and speaker honoraria of Amicus Therapeutics, Sanofi-Genzyme and Shire and is an advisory board member of Amicus Therapeutics, Sanofi-Genzyme and Shire. 


\section{Cellular Physiology Cell Physiol Biochem 2019;52:1139-1150 \begin{tabular}{l|l} 
DOI: 10.33594/000000077 & (c) 2019 The Author(s). Published by
\end{tabular} and Biochemistry Published online: 17 April $2019 \quad$ Cell Physiol Biochem Press GmbH\&Co. KG \\ Braun et al.: Enzyme Replacement Therapy in a Podocyte Cell Culture Model of Fabry \\ Disease}

\section{References}

1 Germain DP: Fabry disease. Orphanet J Rare Dis 2010;5:30.

-2 Schiffmann R: Fabry disease. Handb Clin Neurol 2015;132:231-248.

-3 Wanner N, Hartleben B, Herbach N, Goedel M, Stickel N, Zeiser R, Walz G, Moeller MJ, Grahammer F, Huber TB: Unraveling the Role of Podocyte Turnover in Glomerular Aging and Injury. J Am Soc Nephrol 2014:25:707-716.

-4 Eng DG, Kaverina NV, Schneider RRS, Freedman BS, Gross KW, Miner JH, Pippin JW, Shankland SJ: Detection of renin lineage cell transdifferentiation to podocytes in the kidney glomerulus with dual lineage tracing. Kidney Int 2018;93:1240-1246.

-5 Eng CM, Guffon N, Wilcox WR, Germain DP, Lee P, Waldek S, Caplan L, Linthorst GE, Desnick RJ: International Collaborative Fabry Disease Study Group: Safety and efficacy of recombinant human alphagalactosidase A--replacement therapy in Fabry's disease. N Engl J Med 2001;345:9-16.

-6 Schiffmann R, Kopp JB, Austin HA, Sabnis S, Moore DF, Weibel T, Balow JE, Brady RO: Enzyme replacement therapy in Fabry disease: a randomized controlled trial. JAMA 2001;285:2743-2749.

7 Taguchi A, Maruyama H, Nameta M, Yamamoto T, Matsuda J, Kulkarni AB, Yoshioka H, Ishii S: A symptomatic Fabry disease mouse model generated by inducing globotriaosylceramide synthesis. Biochem J 2013;456:373-383.

- 8 Maruyama H, Taguchi A, Nishikawa Y, Guili C, Mikame M, Nameta M, Yamaguchi Y, Ueno M, Imai N, Ito Y, Nakagawa T, Narita I, Ishii S: Medullary thick ascending limb impairment in the GlatmTg(CAG-A4GALT) Fabry model mice. FASEB J;32:4544-4559.

-9 Ohshima T, Murray GJ, Swaim WD, Longenecker G, Quirk JM, Cardarelli CO, Sugimoto Y, Pastan I, Gottesman MM, Brady RO, Kulkarni AB: alpha-Galactosidase A deficient mice: a model of Fabry disease. Proc Natl Acad Sci USA 1997;94:2540-2544.

10 Miller JJ, Aoki K, Moehring F, Murphy CA, O’Hara CL, Tiemeyer M, Stucky CL, Dahms NM: Neuropathic pain in a Fabry disease rat model. JCI Insight 2018;3:pii:99171.

-11 Liebau MC, Braun F, Höpker K, Weitbrecht C, Bartels V, Müller RU, Brodesser S, Saleem MA, Benzing T, Schermer B, Cybulla M, Kurschat CE: Dysregulated autophagy contributes to podocyte damage in Fabry's disease. PLoS One 2013;8:e63506.

12 Kusiak JW, Quirk JM, Brady RO: Purification and properties of the two major isozymes of alphagalactosidase from human placenta. J Biol Chem 1978;253:184-190.

13 Behrends C, Sowa ME, Gygi SP, Harper JW: Network organization of the human autophagy system. Nature 2010;466:68-76.

-14 Sanchez-Niño MD, Sanz AB, Carrasco S, Saleem MA, Mathieson PW, Valdivielso JM, Ruiz-Ortega M, Egido J, Ortiz A: Globotriaosylsphingosine actions on human glomerular podocytes: implications for Fabry nephropathy. Nephrology Dialysis Transplantation 2011;26:1797-1802.

- 15 Sanchez-Niño MD, Carpio D, Sanz AB, Ruiz-Ortega M, Mezzano S, Ortiz A: Lyso-Gb3 activates Notch1 in human podocytes. Hum Mol Genet 2015;24:5720-5732.

-16 Williams SA, McConkey DJ: The proteasome inhibitor bortezomib stabilizes a novel active form of p53 in human LNCaP-Pro5 prostate cancer cells. Cancer Res 2003;63:7338-7344.

-17 Fan JQ Ishii S, Asano N, Suzuki Y: Accelerated transport and maturation of lysosomal alpha-galactosidase A in Fabry lymphoblasts by an enzyme inhibitor. Nature Medicine 1999;5:112-115.

18 Mauer M, Sokolovskiy A, Barth JA, Castelli JP, Williams HN, Benjamin ER, Najafian B: Reduction of podocyte globotriaosylceramide content in adult male patients with Fabry disease with amenable GLA mutations following 6 months of migalastat treatment. J Med Genet 2017;54:781-786.

19 Chévrier M, Brakch N, Céline L, Genty D, Ramdani Y, Moll S, Djavaheri-Mergny M, Brasse-Lagnel C, Annie Laquerrière AL, Barbey F, Bekri S: Autophagosome maturation is impaired in Fabry disease. Autophagy 2010;6:589-599.

20 Palmieri M, Impey S, Kang H, di Ronza A, Pelz C, Sardiello M, Ballabio A: Characterization of the CLEAR network reveals an integrated control of cellular clearance pathways. Hum Mol Genet 2011;20:3852-3866.

21 Sardiello M, Palmieri M, di Ronza A, Medina DL, Valenza M, Gennarino VA, Di Malta C, Donaudy F, Embrione V, Polishchuk RS, Banfi S, Parenti G, Cattaneo E, Ballabio A: A gene network regulating lysosomal biogenesis and function. Science 2009;325:473-477. 


\section{Cellular Physiology Cell Physiol Biochem 2019;52:1139-1150 \begin{tabular}{l|l}
\hline DOI: 10.33594/000000077 & (c) 2019 The Author(s). Published by
\end{tabular} and Biochemistry Published online: 17 April $2019 \quad$ Cell Physiol Biochem Press GmbH\&Co. KG \\ Braun et al.: Enzyme Replacement Therapy in a Podocyte Cell Culture Model of Fabry \\ Disease}

-22 Spampanato C, Feeney E, Li L, Cardone M, Lim JA, Annunziata F, Zare H, Polishchuk R, Puertollano R, Parenti G, Ballabio A, Raben N: Transcription factor EB (TFEB) is a new therapeutic target for Pompe disease. EMBO Mol Med 2013;5:691-706.

-23 Song W, Wang F, Savini M, Ake A, di Ronza A, Sardiello M, Segatori L: TFEB regulates lysosomal proteostasis. Hum Mol Genet 2013;22:1994-2009.

24 Alghamdi TA, Majumder S, Thieme K, Batchu SN, White KE, Liu Y, Brijmohan AS, Bowskill BB, Advani SL, Woo M, Advani A: Janus Kinase 2 Regulates Transcription Factor EB Expression and Autophagy Completion in Glomerular Podocytes. J Am Soc Nephrol 2017;28:2641-2653.

25 Slaats GG, Braun F, Hoehne M, Frech LE, Blomberg L, Benzing T, Schermer T, Rinschen MM, Kurschat CE: Urine-derived cells: a promising diagnostic tool in Fabry disease patients. Sci Rep 2018;8:11042.

26 Saleem MA, O’Hare MJ, Reiser J, Coward RJ, Inward CD, Farren T, Xing CY, Ni L, Mathieson PW, Mundel P: A conditionally immortalized human podocyte cell line demonstrating nephrin and podocin expression. J Am Soc Nephrol 2002;13:630-638.

27 Mehta A, Beck M, Elliott P, Giugliani R, Linhart A, Sunder-Plassmann G, Schiffmann R, Barbey F, Ries M, Clarke JT, Fabry Outcome Survey investigators: Enzyme replacement therapy with agalsidase alfa in patients with Fabry's disease: an analysis of registry data. Lancet 2009;374:1986-1996.

28 Germain DP, Charrow J, Desnick RJ, Guffon N, Kempf J, Lachmann RH, Lemay R, Linthorst GE, Packman S, Scott CR, Waldek S, Warnock DG, Weinreb NJ, Wilcox WR: Ten-year outcome of enzyme replacement therapy with agalsidase beta in patients with Fabry disease. J Med Genet 2015;52:353-358.

29 Hughes DA, Nicholls K, Shankar SP, Sunder-Plassmann G, Koeller D, Nedd K, Vockley G, Hamazaki T, Lachmann R, Ohashi T, Olivotto I, Sakai N, Deegan P, Dimmock D, Eyskens F, Germain DP1 Goker-Alpan O, Hachulla E, Jovanovic A, Lourenco CM: Oral pharmacological chaperone migalastat compared with enzyme replacement therapy in Fabry disease: 18-month results from the randomised phase III ATTRACT study. J Med Genet 2017;54:288-296.

30 Rozenfeld P, Feriozzi S: Contribution of inflammatory pathways to Fabry disease pathogenesis. Molecular Genetics and Metabolism 2017;122:19-27.

31 Lavoie P, Boutin M, Abaoui M, Auray-Blais C: Fabry Disease Biomarkers: Analysis of Urinary Lyso-Gb3 and Seven Related Analogs Using Tandem Mass Spectrometry. Curr Protoc Hum Genet 2016;90:17.22.117.22.12.

- 32 Tortorelli S, Turgeon CT, Gavrilov DK, Oglesbee D, Raymond KM, Rinaldo P, Matern D: Simultaneous Testing for 6 Lysosomal Storage Disorders and X-Adrenoleukodystrophy in Dried Blood Spots by Tandem Mass Spectrometry. Clinical Chemistry 2016;62:1248-1254.

33 Pettazzoni M, Froissart R, Pagan C, Vanier MT, Ruet S, Latour P, Guffon N, Fouilhoux A, Germain DP, Levade T, Vianey-Saban C, Piraud M, Cheillan D: LC-MS/MS multiplex analysis of lysosphingolipids in plasma and amniotic fluid: A novel tool for the screening of sphingolipidoses and Niemann-Pick type C disease. PLoS One 2017;12:e0181700.

- 34 Tøndel C, Bostad L, Hirth A, Svarstad E: Renal biopsy findings in children and adolescents with Fabry disease and minimal albuminuria. Am J Kidney Dis 2008;51:767-776.

- 35 Tøndel C, Kanai T, Larsen KK, Ito S, Politei JM, Warnock DG, Svarstad E: Foot Process Effacement Is an Early Marker of Nephropathy in Young Classic Fabry Patients without Albuminuria. Nephron Physiol 2014;129:16-21.

36 Svarstad E, Leh S, Skrunes R, Kampevold Larsen K, Eikrem Ø, Tøndel C: Bedside Stereomicroscopy of Fabry Kidney Biopsies: An Easily Available Method for Diagnosis and Assessment of Sphingolipid Deposits. Nephron 2017;138:13-21.

37 Jamboti J, Forrest CH: Fabry disease; early diagnosis improves prognosis but diagnosis is often delayed. J Nephropathol 2017;6:130-133.

- 38 Arends M, Biegstraaten M, Hughes DA, Mehta A, Elliott PM, Oder D, Watkinson OT, Vaz FM, van Kuilenburg ABP, Wanner C, Hollak CEM: Retrospective study of long-term outcomes of enzyme replacement therapy in Fabry disease: Analysis of prognostic factors. PLoS ONE 2017;12:e0182379. 